\title{
Observations on a Giant Sigmoid Volvulus with Unusual Clinical Presentation and Challenging Postoperative Course: Case Report
}

\author{
Giuseppe Pisano*, Pietro Giorgio Calò, Stefano Piras, Enrico Erdas \\ Department of Surgical Sciences, University of Cagliari, Cagliari, Italy \\ Email: "gpisano@unica.it
}

Received 21 September 2015; accepted 10 November 2015; published 13 November 2015

Copyright (C) 2015 by authors and Scientific Research Publishing Inc.

This work is licensed under the Creative Commons Attribution International License (CC BY). http://creativecommons.org/licenses/by/4.0/

(c) (i) Open Access

\begin{abstract}
Introduction: The present report describes a case of a giant sigmoid volvulus (SV) where acute respiratory distress was associated with toxic megacolon. Clinical features, surgical treatment and postoperative course deserved our attention and discussion. Presentation of Case: A 67-year-old man with psychiatric disturbances was admitted to our Department with severe respiratory distress due to an enormous abdominal distension caused by a sigmoid volvulus. Endoscopic derotation was unsuccessful and surgery immediately performed. After a wide colonic resection the patient underwent a prolonged treatment in the Intensive Care Unit. Death occurred 34 days after the operation for secondary infection of peritoneal effusion. Discussion: Main clinical features of SV pertain to abdominal compartment while in the present case acute respiratory distress was the prominent symptom; in the same time the severity of the case was due to the association of high abdominal pressure together with a toxic megacolon. Postoperative treatment consisted in ventilatory support, with a progressive shift from asssisted to spontaneous ventilation; repeated sessions of haemodialysis were necessary to manage renal failure up to recovery of the urine output. Bacterial trans location due to toxic megacolon was responsible of late infection of ascitic fluid. In spite of multiple antibiotic association according to bacterial cultures, intra-abdominal abscesses eventually developed causing fatal outcome 34 days after the first intervention. Conclusion: Severe clinical presentation required a prolonged and demanding postoperative course which was focused on the recovery of respiratory, cardiac and renal function even if fatal outcome was due to septic complications. Suspicion of late infection of ascitic fluid could arise from persistently high values of inflammation indexes and drive to an earlier drainage of the abdominal abscesses.
\end{abstract}

\footnotetext{
${ }^{*}$ Corresponding author.
} 


\section{Keywords}

\section{Sigmoid Volvulus, Toxic Megacolon, Abdominal Compartment Syndrome}

\section{Introduction}

Common complaints of sigmoid volvulus (SV) are usually represented by sudden abdominal pain, distension and arrested flatus passage, while physical examination reveals abdominal tenderness and asymmetrical abdominal distension [1]-[4]. If these are the most frequent findings, sometimes clinical presentation may change towards a frank peritonitic syndrome or an abdominal compartment syndrome (ACS), where the increased abdominal pressure compromises the functions of visceral organs [2] [5]-[8].

Association of SV and ACS is not uncommon, but their occurrence together with severe respiratory distress and advanced colonic ischemia is seldom found. The present paper describes a case where an acute SV presented with such a massive distension to cause a severe respiratory distress and renal flow impairment; in the same time colonic ischemia due to bowel strangulation was already responsible of a condition evolving towards a septic shock.

The unusual clinical presentation and the challenging postoperative course made this case worth of the present report.

\section{Case Presentation}

A 66-year-old man with severe psychotic disturbances was admitted to our Ward from the First Aid Department with a diagnosis of "Megacolon, colicky abdominal pain and bowel obstruction". At the time of admission the patient was pale, sweating, in shortness of breath, with a Blood Pressure of 100/70, Heart Rate 106, O2 Sat 84\%; his lower extremities were cold, subcyanotic and moreover he presented an enormous abdominal distension (Figure 1).

From the accompanying persons we were informed that a similar episode occurred few weeks before and was successfully treated with cleansing enemas; his last bowel movements occurred 10 days before.

Resuscitation with cristalloid infusion was immediately started, anaesthesiologists and cardiologists were called for assistance; oxygen was administered through nasal devices. Heart evaluation and Echocardiogram were unsuccessful. After insertion of a catheter and a naso-gastric tube, the patient was taken to the Radiology Unit for a CT: initial Plain Abdomen showed an enormous colonic distension with severe elevation of diaphragm and a shift to the right of the cardiomediastinal profile (Figure 2).

Axial pictures confirmed an enormous colonic distension due to a SV extending from the pelvis up to the thorax nearly at the level of the tracheal bifurcation (Figure 3).

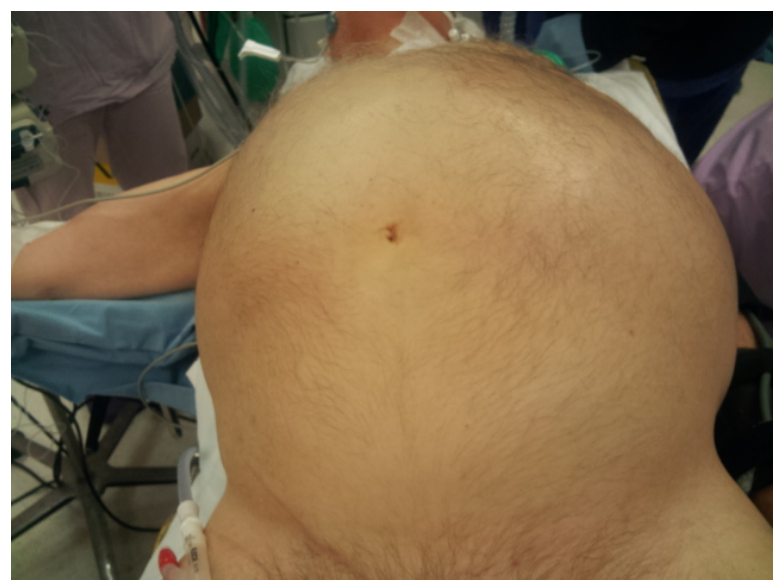

Figure 1. Patient on the operation table: clinical appearance of the huge abdominal distension. 


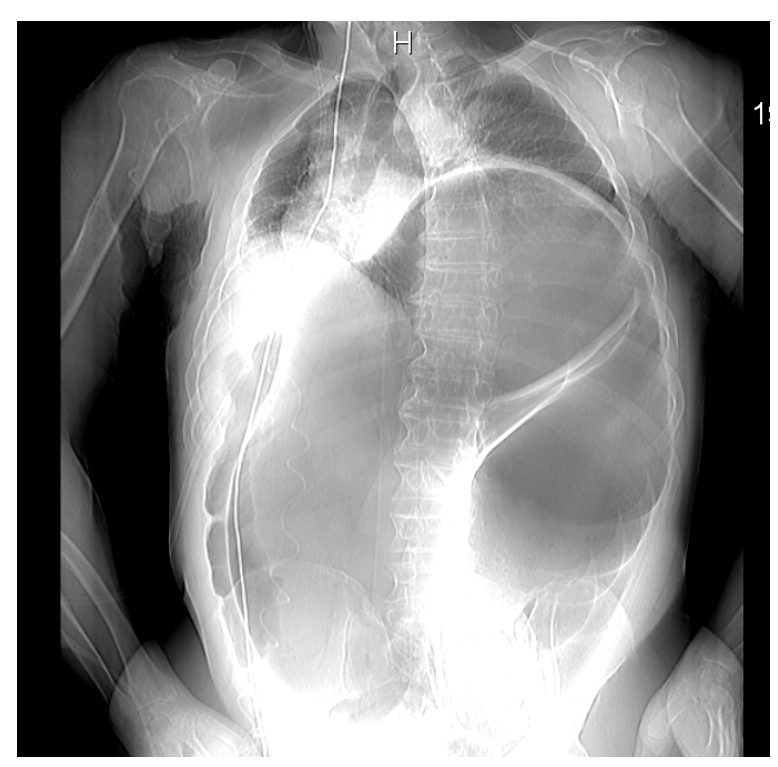

Figure 2. Plain X Ray abdomen: diaphragm elevation, lungs compression and right mediastinal shift due to the sigmoid distension.

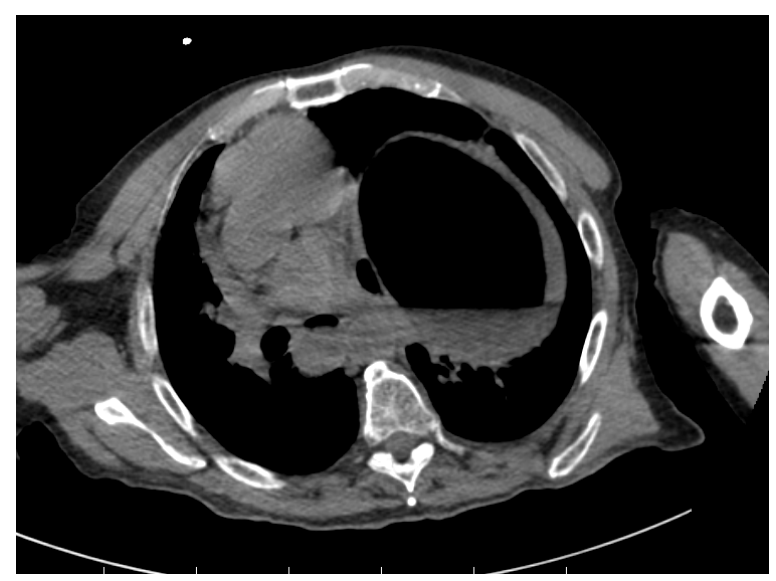

Figure 3. Upper CT scan showing the sigmoid colon reaching the level of tracheal bifurcation and mediastinum moved to the right.

Just after CT the patient was taken to the operating room: an endoscopic attempt of colonic decompression was abandoned because of the finding of ischaemic features of the colonic mucosa. A median laparotomy showed an enormous distension of sigmoid colon which looked like an inflated inner tube of a middle size car (Figure 4).

Surgical procedures consisted in a wide colosigmoid resection (Figure 5), drainages and terminal colostomy.

After surgery the patient was transferred to the Intensive Care Unit (ICU) for assisted ventilation; haemodialysis was started in Post Operative Day (POD) 3 because of anuria since the operation; in the same day a colonoscopic was performed to exclude ischaemia of the remaining large bowel; repeated blood transfusions were necessary for persistent anaemia.

Bronchoalveolar lavage was positive for Staphilococcus aureus (POD 1 and 3) while pseudomonas aeruginosa was detected on the first drainage tube removed from the abdomen (POD 7); candida colonies were found in bronchial secretion the same day. Antibiotic therapy was modified according to microbiologic findings, first association of imipenem, teicoplanin and fluconazole was changed with a second combination of ticarcillin with clavulanic acid, levofloxacin and fluconazole and with a latest association of amikacin with meropenem. 


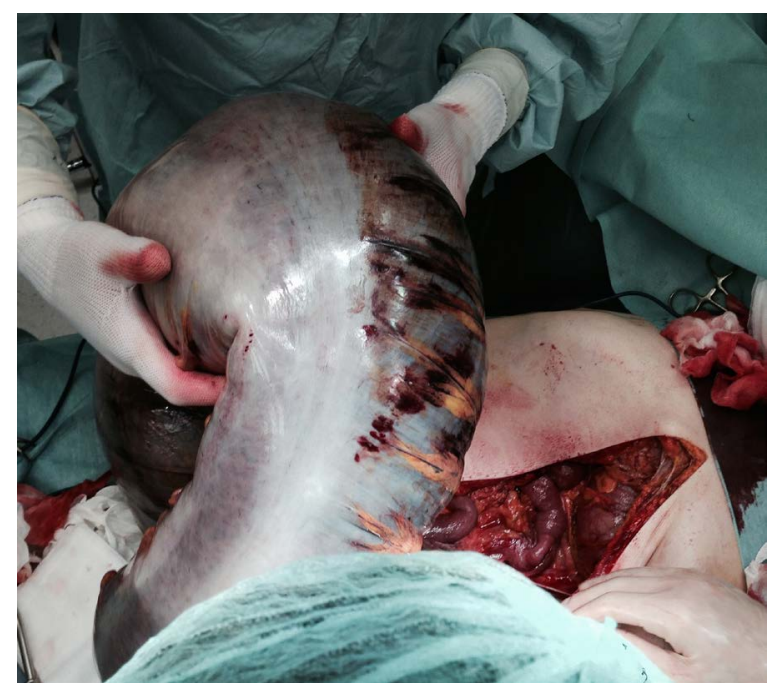

Figure 4. Intraoperative picture: sigmoid colon looks like an inner tube of a middle size car.

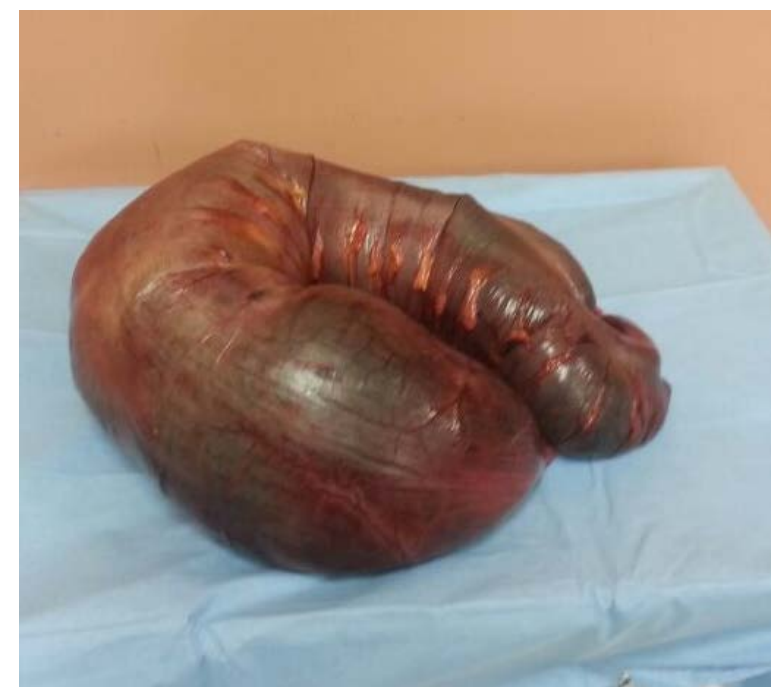

Figure 5. Operative specimen of colonic resection.

Prolonged ascitic effusion came out through the other pelvic drainage since POD 22: two days later the tube was removed when no more liquid escaped. Tracheal tube was taken in POD 16 and since then the patient was in spontaneous breathing with mask oxygen administration.

During the final stay in ICU however, levels of procalcitonin and presepsin remained constantly high as well as $\mathrm{WBC}$ and neutrophils percentage.

The patient returned to our ward in the 28th POD, breathing spontaneously, with normal diuresis but still febrile. Within a few hours since the admission an abundant, purulent like discharge started to flow from the colostomy edges with characters very similar to enteric fluid. A new CT scan revealed two large purulent communicating collections in the right and left paracolic gutters (Figure 6): in spite of percutaneous CT guided drainage, pus evacuation was not satisfactory. Such condition, together with the suspicion of an enteric leakage brought to a new laparotomy: no enteric leakage was found, the two collections were evacuated and after peritoneal toilet three new drainages were left in the abdominal cavity. During the second postoperative course the patient underwent further respiratory distress, recurrent renal failure and required two new blood transfusions. The patient died while asleep in the 5th POD (34 days after the primary operation). Autopsy was not performed. 


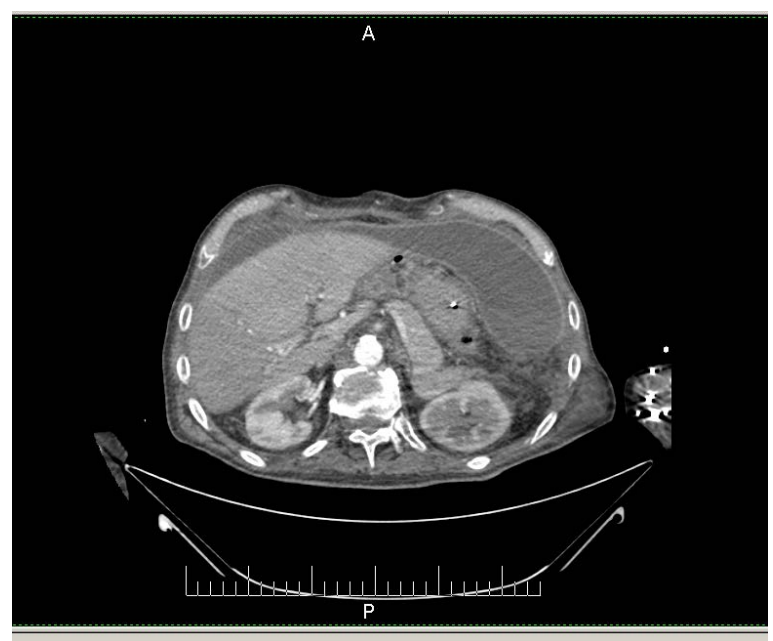

Figure 6. Late postoperative CT scan showing two large communicating purulent collections along the right and left paracolic gutter.

\section{Discussion}

Main symptoms of SV differ significantly between endemic and sporadic forms [2], but in the majority of cases abdominal distension, pain and constipation are the leading presenting features of such disease [1] [3]-[5]. In the present case the prominent clinical finding was an acute respiratory distress due to diaphragm compression and mediastinum shift to the right: such occurrence is rarely described [2] and in the present report could be diagnosed only after CT scan. It is not an accident if cardiologist were not able to give any information on heart function because ultrasonography could not detect the usual echographic findings.

Recognition of the severity of SV came then only after CT scan and the patient taken to the operating room where ventilatory support was shortly established. Surgery was performed after an initial attempt of endoscopic decompression. On such item it is a common agreement that SV should undergo to an endoscopic approach as a first option (unless signs of peritonitis are already present) and surgery performed as a second step in case of failed endoscopy [1] [3]-[5] [9]

The preferred surgical choice in emergency is usually a resection of sigmoid loop with an end colostomy, the so called Hartmann procedure [10]-[12] which was actually the operation performed in the present case. Some Authors describe sigmoid resection and primary anastomosis [9] [12] [13], less frequent operations include colopexy, mesosigmoidoplasty but such procedures are mainly performed in elective conditions [9] [14].

In our case emergency surgery required a wide colonic resection because after removal of the huge necrotic sigmoid loop, another segment of descending colon presented the signs of bowel ischemia. Safety of colonic viability could not be reached at that time also because pathologic condition was that of a toxic megacolon with extensive microbial translocation together with microvessels thrombosis. Bhatnagar et al. found in $26 \%$ of their series that colonic ischemia extended beyond the area of constriction into the rectum/descending colon: such extension may have an ill defined line of demarcation and could lead to an error in judgement of bowel viability [11].

The possible adoption of a laparostomy in the present case was considered but not adopted because resection of the long dilated ischemic colon, together with terminal colostomy, appeared good enough in removing the main cause of high intra-abdominal pressure. Review of the recent literature shows that laparostomy is mainly adopted in cases of severe peritonitis to control predictable occurrence of further peritoneal infections [15] [16]. According to the cited references of the present work laparostomy was never performed after surgery for SV.

Overall mortality for SV ranges from $10 \%$ to $55 \%$, higher values are reported for emergency operations and depend on the presence of bowel gangrene, peritonitis, age and associated diseases [2] [11] [13].

In our case we were really expecting a fatal outcome since the very few days after operation; in the meanwhile medical attention was addressed to restore pulmonary ventilation and acute renal failure as well as prevention of microbial infections. When all these conditions looked at last obtained the patient was transferred 
from the ICU to our ward.

At the readmission, 4 weeks after surgery, the patient was in spontaneous breathing, good circulating conditions and normal urine output, whereas his leukocytes count and inflammation markers kept high since several days. The improving general conditions during the ICU stay reduced the attention on high leukocytes values as well as procalcitonin and presepsin markers; no suspicion of a latent abdominal infection arose from these indexes, also because of absence of fever and normal bowel movement from the colonic stoma. Latest attempt to drain purulent collections could not prevent an immanent condition of septic shock.

It is questionable if an earlier diagnosis could have prompted an effective therapy: the lesson from this case however obliges a long lasting attention on late postoperative infections even when other organs function had already recovered.

\section{Conclusions}

Together with the unusual clinical presentation, the reported case of SV was responsible of severe impairment of cardiac, lung and renal functions: such condition required the attention of the intensive care operators aimed to restore multiple organ impairment. Late infection of ascitic fluid did occur when good ventilation and urinary function were finally accomplished and the patient seemed overcoming the critical phase. Persistence of laboratory signs of sepsis should have prompted an earlier abdominal CT to discover the purulent collections.

Suspicion of late infections in such case could have made surgery performed at an earlier stage.

Conclusion dictates that in critical patients a late septic complication can not be excluded even when good organs function has been obtained.

\section{References}

[1] Atamanalp, S.S. (2013) Sigmoid Volvulus: Diagnosis in 938 Patients over 45.5 Years. Techniques in Coloproctology, 17, 419-424. http://dx.doi.org/10.1007/s10151-012-0953-z

[2] Raveenthiran, V., Madiba, T.E., Atamanalp, S.S. and De, U. (2010) Volvulus of the Sigmoid Colon. Colorectal Disease, 12, e1-e17. http://dx.doi.org/10.1111/j.1463-1318.2010.02262.x

[3] Gingold, D. and Murrell, Z. (2012) Management of Colonic Volvulus. Clinics in Colon and Rectal Surgery, 25, 236244. http://dx.doi.org/10.1055/s-0032-1329535

[4] Chung, Y.F.A., Eu, K.-W., Nyam, D.C.N.K., Leong, A.F.P.K., Ho, Y.H. and Seow-Choen, F. (1999) Minimizing Recurrence after Sigmoid Volvulus. British Journal of Surgery, 86, 231-233. http://dx.doi.org/10.1046/j.1365-2168.1999.01034.x

[5] Lou, Z., Yu, E.-D., Zhang, W., Meng, R.-G., Hao, L.-Q. and Fu, C.-G. (2013) Appropriate Treatment of Acute Sigmoid Volvulus in the Emergency Setting. World Journal of Gastroenterology, 19, 4979-4983. http://dx.doi.org/10.3748/wjg.v19.i30.4979

[6] Meldrum, D.R., Moore, F.A., Moore, E.E., Franciose, R.J., Sauaia, A. and Burch, J.M. (1997) Prospective Characterization and Selective Management of the Abdominal Compartment Syndrome. The American Journal of Surgery, 174, 667-673. http://dx.doi.org/10.1016/S0002-9610(97)00201-8

[7] Diebel, L.N., Dulchavsky S.A. and Brown W.J. (1997) Splanchnic Ischemia and Bacterial Translocation in the Abdominal Compartment Syndrome. The Journal of Trauma, 43, 852-855.

[8] Decker, G. (2001) Abdominal Compartment Syndrome. Journal de Chirurgie, 138, 270-276.

[9] Safioleas, M., Chatzconstatinou, C., Felekouras, E., Stamatakos, M., Papaconstantinou, I., Smirnis, A., Safioleas, P. and Kostakis, A. (2007) Clinical Considerations and Therapeutic Strategies for Sigmoid Volvulus in the Elderly: A Study of 33 Cases. World Journal of Gastroenterology, 13, 921-924. http://dx.doi.org/10.3748/wjg.v13.i6.921

[10] Gupta, T.K., Jha, J.K., Biswas, R.S., Chattopadhyay, S.D., Gupta, N.K., Kumar, S., Nath, N.C. and Karmakar, N.C. (2011) Emergency Management of Sigmoid Volvulus - Institutional Experience over Four Years. Journal of the Indian Medical Association, 109, 714-716.

[11] Bhatnagar, B.N., Sharma, C.L, Gautam, A., Kakar, A. and Reddy, D.C. (2004) Gangrenous Sigmoid Volvulus: A Clinical Study of 76 Patients. International Journal of Colorectal Disease, 19, 134-142. http://dx.doi.org/10.1007/s00384-003-0534-8

[12] Kuzu, M.A., Aslar, A.K., Soran, A., Polat, A., Topcu, O. and Hengirmen, S. (2002) Emergent Resection for Acute Sigmoid Volvulus. Results of 106 Consecutive Cases. Diseases of the Colon \& Rectum, 45, 1085-1090. http://dx.doi.org/10.1007/s10350-004-6364-0 
[13] Halabi, W.J., Jafari, M.D., Kang, C.Y., Nguyen, V.Q., Carmichael, J.C., Mills, S., Pigazzi, A. and Stamos, M.J. (2014) Colonic Volvulus in the United States: Trends, Outcomes, and Predictors of Mortality. Annals of Surgery, 259, $293-$ 301. http://dx.doi.org/10.1097/SLA.0b013e31828c88ac

[14] Cirocchi, R., Farinella, E., La Mura, F., Morelli, U., Trastulli, S., Milani, D., Di Patrizi, M.S., Rossetti, B., Spizzirri, A., Galanou, I., Kopanakis, K., Mecarelli, V. and Sciannameo, F. (2010) The Sigmoid Volvulus: Surgical Timing and Mortality for Different Clinical Types. World Journal of Emergency Surgery, 5, 1. http://dx.doi.org/10.1186/1749-7922-5-1

[15] Bailey, C.M., Thompson-Fawcett, M.W., Kettlewell, M.G., Garrard, C. and Mortensen, N.J. (2000) Laparostomy for Severe Intra-Abdominal Infection Complicating Colorectal Disease. Diseases of the Colon \& Rectum, 43, 25-30. http://dx.doi.org/10.1007/BF02237239

[16] Anderson, O., Putnis, A., Bhardway, R,. Ho-Asjoe, M., Carapeti, E., Williams, A.B. and George, M.L. (2011) Shortand Long-Term Outcome of Laparostomy Following Intra-Abdominal Sepsis. Colorectal Disease, 13, e20-e32. http://dx.doi.org/10.1111/j.1463-1318.2010.02441.x 